\title{
Characterization of X-Ray Diamond Detector by Monte Carlo Method
}

\author{
S.J. Stankovic*, R.D. Ilic, D.M. Davidovic and M. Petrovic \\ Vinca Institute of Nuclear Science, Radiation and Environmental Protection Laboratory \\ P.O. Box 522, 11001 Belgrade, Serbia
}

\begin{abstract}
The use of diamond as material for X-ray detector is subject of investigation and practice in radiotherapy, space and material science and technology. This paper presents the results of application of Monte Carlo method for simulation of photon transport through diamond detector. The aim is restitution and demonstrating of numerical technique for characterization of electrical properties for different detector conditions and configurations. Monte Carlo code was adopted to determine the energy deposited and dose distribution in the structure of diamond detector. Our results show that the use of numerical simulations may be of essential help in design of diamond detector systems.
\end{abstract}

PACS numbers: 29.40.Wk

\section{Introduction}

In the modern era the practice of experimental particle physics usually requires the use of extremely large and costly composite detectors. The very promising area of application for diamond is in the construction of these detectors, where it is much greater resistance to radiation damage, and several of its other physical properties make it extremely attractive together with the established technology based on silicon [1]. In order to make it possible to contemplate the use of diamond in such detectors, it is necessary to obtain, at reasonable cost, thin sheets of diamond of millimeter dimensions. The development of the chemical vapor deposition (CVD) process for diamond manufacture, with attendant substantial costs of the large and complicated plant required for industrial-scale exploitation, has been driven by the requirements, not of particle physics detectors, for which the potential market is relatively small, but of other applications which exploit the remarkable properties of diamond in other ways. The optimization of CVD diamond manufacture may not yield material well suited to detector applications, but there is also a large potential market for electronic devices such as diodes and transistors using diamond as the active medium, and here there is more in common with requirements for detector diamond.

X-ray dosimetry with new detecting materials is a very interesting field of research not only for radiotherapy and radiology, but also for space technology. The recent availability of CVD diamond single crystals obtained by homoepitaxy on diamond, which can display electronic properties even superior to those of natural diamond,

\footnotetext{
* corresponding author; e-mail: srbas@vinca.rs
}

may open new real possibilities for X-ray dosimetry and monitoring X-ray pulses delivered by a portable X-ray apparatus [2].

Detectors based on diamond have been evaluated in photon and electron beams and show great promise as an alternative to ionization chambers and Si diodes. Small detectors can be made, as for diodes, with good spatial resolution. The atomic number of diamond is 6 compared to 7.4 for soft tissue and so nearly tissue equivalent, removing the need for energy dependent corrections to the detector signal. Initially, the signals were very small but as processing has improved this has led to devices with sufficiently good signal to noise ratio to be used as tracking detectors [3]. A diamond detector type (PTW Freiburg) was examined for the purpose of dosimetry with 4-20 MeV electron and 4-25 MeV photon beams. Results were compared with those obtained by using a Markus chamber for electron beams and ionization chamber for photon beams. The superior spatial resolutions of the diamond detector leads to minor deviations between depth-dose curves of electron beams measured with a Markus chamber and a diamond detector. Depth-dose curves of photon beams, measured with the diamond detector, show a slight overestimation compared with measurements with the ionization chamber [4]. Detectors based on polycrystalline diamonds seem to be best candidates for future dosimetry, since they have most of the characteristics of natural diamonds at potentially much lower cost. In this context, the performances of laboratory made polycrystalline diamond device are compared to those of the three, PTW, Scanditronix and Thomson commercial dosimeters [5].

The thickness of any given material where $50 \%$ of the incident energy has been attenuated is know as the halfvalue layer (HVL). Polycrystalline diamond detectors 
with energy resolving capability of the impinging beam were realized and tested by using a miniature pyroelectric X-ray pulse generator [6]. The response of a commercially available diamond detector (PTW Freiburg/IPTB Dubna) at $45 \mathrm{kVp}(0.55 \mathrm{~mm} \mathrm{Al} \mathrm{first} \mathrm{HVL)} \mathrm{and} 100 \mathrm{kVp}$ (2.3 $\mathrm{mm} \mathrm{Al} \mathrm{first} \mathrm{HVL)} \mathrm{including} \mathrm{dose} \mathrm{and} \mathrm{dose-rate}$ linearity, percentage depth-dose and output factors is a function of applicator size. Comparisons are made with data of measurements using a PTW parallel-plate chamber, diamond detector and Monte Carlo simulations based on spectra determined from transmission measurements in aluminium. Excellent agreement was obtained for percentage depth-dose curves between Monte Carlo and diamond after correcting for sublinearity of the dose-rate response and energy dependence of the diamond detector. However, significant differences were noted between diamond detector/Monte Carlo and the parallel-plate chamber, which is attributed to the perturbation caused by the polyethylene base of the chamber [7].

Owing to the problems recognized in practice, the aim of our paper is to determine the distribution of the energy deposited in a diamond wafer of detector using computer simulation based on the Monte Carlo techniques. Advanced computer program FOTELP-2K6 [8] was used for this purpose. The obtained results provided conclusions regarding the possibilities of applied code in analysing diamond detector characteristics in radiation fields.

\section{Theory}

Photons of $\mathrm{X}$ and gamma rays, being uncharged, do not lose energy continuously in matter as charged particle do, and hence produce no signal until they generate a free electron in the material. For incident radiation at least a few times above the band gap, the average energy to create one electron-hole pair $\left(E_{\text {eh }}\right)$ in diamond is known to be $13 \mathrm{eV}$ [9]. The electron-hole pairs that are generated will be swept apart under the applied field, but will generally trap before reaching the contacts. The average separation distance $(d)$ of an electron-hole pair is

$$
d=\left(\mu_{\mathrm{e}} \tau_{\mathrm{e}} F+\mu_{\mathrm{h}} \tau_{\mathrm{h}} F\right) \propto 1 / N_{\mathrm{t}},
$$

where $\mu_{\mathrm{e}, \mathrm{h}}$ is the electron/hole mobility, $\tau_{\mathrm{e}, \mathrm{h}}$ is the electron/hole lifetime, $F$ is the applied electric field and $N_{\mathrm{t}}$ is the concentration of trapping sites in the crystal. Since $d$ is inversely proportional to the trapping site concentration, its value is commonly taken as an indicator of the electrical quality of a crystal (a higher value of $d$ indicating better quality). We can determine the value of $d$ for a given sample of thickness, $A$, by using the following approximate relation (valid in the region $d \ll A$ ):

$$
d \approx\left(Q_{\mathrm{M}} / Q_{\mathrm{T}}\right) A,
$$

where $Q_{\mathrm{T}}$ is the total charge liberated by the incident radiation and $Q_{\mathrm{M}}$ is the total induced charge that is actually measured by integrating the current signal at the scope. It can be shown that while $Q_{\mathrm{T}}$ is implicitly dependent on detector area $(S)$ and thickness $(A), Q_{\mathrm{M}}$ is implicitly dependent only on $S$ and $F$, but not on $A$.
Therefore, as expected, we see that $d$ will be a function of $F$ but not of $A$.

The value of $Q_{\mathrm{T}}$ (in coulombs) can be determined by the relation

$$
Q_{\mathrm{T}}=\left(E_{\mathrm{DEP}} / E_{\mathrm{eh}}\right)\left[1.6 \times 10^{-19}\right],
$$

where $E_{\mathrm{DEP}}$ is the total energy deposited by incident radiation (a quantity that must be calculated).

\section{Characterization method}

\subsection{Materials and geometry}

In this work, the geometric form of the diamond detector was defined with corresponding software programs [8]. FOTELP-2K6 program uses Rvachev R-functions geometry (RFG), for detector geometry description. According to the available data for diamond detector (Fig. 1), cylindrical diamond wafer has $10 \mathrm{~mm}$ diameter and $1 \mathrm{~mm}$ thickness. The shape of electrical contacts by cooper $(\mathrm{Cu})$ were $8 \mathrm{~mm}$ circles, and $0.1 \mathrm{~mm}$ thickness.

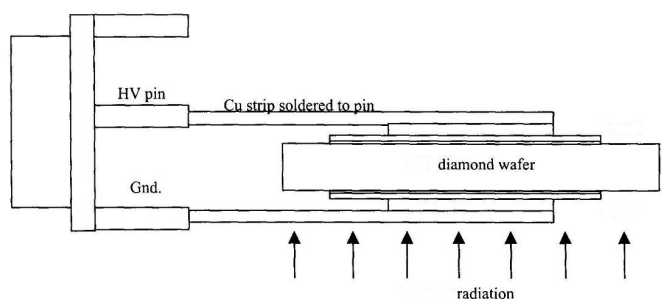

Fig. 1. Schematic diagram of the diamond detector and holder.

\subsection{Numerical method}

Characterization of X-ray diamond detector by Monte Carlo method was made with FOTELP-2K6 code. Physical rigor is maximized by employing the best available cross-sections and high speed routines for random values sampling from their distributions, and the most complete physical model for describing the transport and production of the photon/electron/positron cascade from $100.0 \mathrm{MeV}$ down to $1.0 \mathrm{keV}$. FOTELP-2K6 is developed for numerical experiments by Monte Carlo techniques for dosimetry, radiation damage, radiation therapy and other actual applications of these particles. For the photon history, the trajectory is generated by following it from scattering to scattering using corresponding inverse distribution between collision, types of target, types of collisions, types of secondaries, their energy and scattering angles. Photon interactions are coherent scattering, incoherent scattering, photoelectric absorption and pair production. The Doppler broadening in the Compton scattering is taken. The histories of secondary photons include bremsstrahlung and positron-electron annihilation radiation. The condensed history Monte Carlo method is used for the electron and positron transport simulation. 
During a history, the particles lose energy in collisions, and the secondary particles are generated on the step according to the probabilities for their occurrence. Electron (positron) energy loss is through inelastic electronelectron $\left(\mathrm{e}^{-}, \mathrm{e}^{-}\right)$and positron-electron $\left(\mathrm{e}^{+}, \mathrm{e}^{-}\right)$collisions and bremsstrahlung generation. The fluctuation of energy loss (straggling) is included according to the Landau or Blunk-Westphal distributions with 9 Gaussians. The secondary electrons, which follow history of particles, include knock-on, pair production, Compton and photoelectric electrons. The secondary positrons, which follow pair production, are included, too. With atomic data, the electron and positron Monte Carlo simulation is broadened to treat atomic ion relaxation after photoeffect and impact ionization. Flexibility of the codes permits them to be tailored to specific applications and allows the capabilities of the codes to be extended to more complex applications, especially in radiotherapy in voxelized geometry using computer tomography (CT) data.

\section{Results and discussion}

The general scheme of physical model used in Monte Carlo simulation described in Sect. 3 is applied to the specific geometrical configuration represented in Fig. 1 and for various material layers arrangements. In numerical experiment monoenergetic beam of photons containing $10^{7}$ particles is incoming perpendicularly to the upper surface of the sensitive volume. Photon's energies were $0.1,0.5,1.25,6$, and $10 \mathrm{MeV}$.

All trajectories of the particles within the structural components of detector were monitored: (a) to register deposited energy in material zones, (b) to split the zones into the desired number of layer along the beam axes, and register the values of the deposited energy in the layers of different zones $\mathrm{d} E / \rho \mathrm{d} z\left[\mathrm{MeV} \mathrm{cm}^{2} / \mathrm{g}\right]$. Total deposited photon energies in the detector material zones (diamond wafer and cooper electrodes) are shown in Fig. 2. Distribution of deposited energy in layer of diamond wafer is shown in Fig. 3.

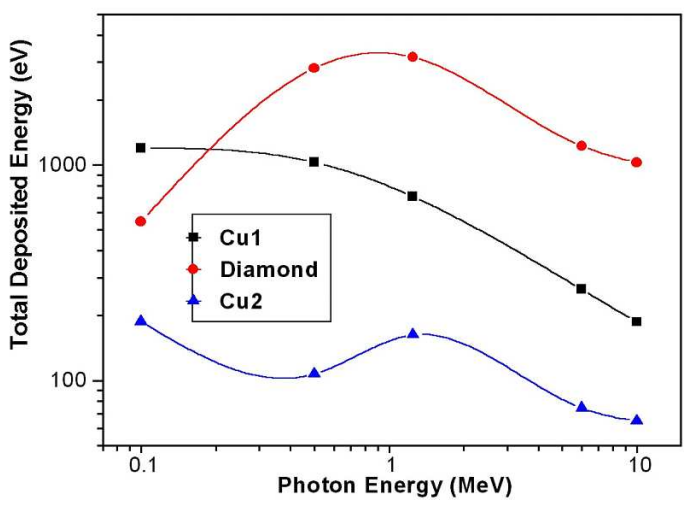

Fig. 2. Total deposited energy in diamond wafer and copper electrodes.

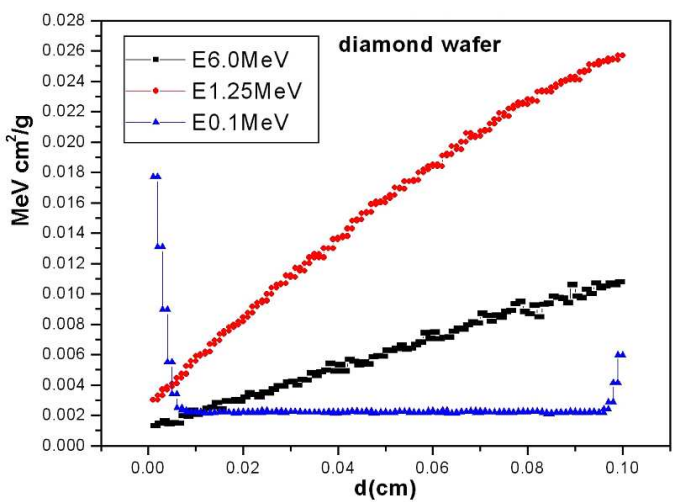

Fig. 3. Deposited energy in layer of diamond wafer.

The most interesting zone in our case is the diamond wafer. From Fig. 2 one can see that in this zone the efficiency of detection decreases with increase in X-ray energy. This fact may cause additional difficulties in detector signal processing in the corresponding electronical system, and must necessarily be taken into account.

Regarding the degree of space homogeneity of the generated electron-hole pairs as a function of energy, the better homogeneity is achieved for lower energies.

Inspecting Fig. 3 one can see that the density in space of the electron-hole pairs increases almost linearly in the direction of the X-ray beam.

\section{Conclusions}

In this paper, numerical experiments were used for the characterization of important parameter for diamond detector fabrication. The results obtained can help one to choose such materials which are of interest for a more detailed experimental investigation. In fact, for each special type of diamond detector one can perform Monte Carlo simulation before experimental investigation in X-ray fields are done in order to obtain in advance sufficiently reliable information about the expected response of the detector with a defined structure. In this way one can choose such a structure which has optimal response for a defined range of energy.

Our intention in this paper was, among other things, to initiate ideas on how to set up appropriate experiments, which would follow conditions imposed by numerical simulations. However, the problem of electrical measurements of properties in medium that has microscopic (or even less) dimensions are well known. Thus, the purpose of this paper is to present the possibilities of the numerical simulations for the deposited energy distribution on microscopic or submicroscopic levels, primarily in qualitative sense. Further investigations related to the study of electrical and technological characteristics of the diamond and related material components are planned. 


\section{References}

[1] R.J. Tapper, Rep. Prog. Phys. 63, 1273 (2000).

[2] A. Balducci, Y. Garino, A. Lo Giudice, C. Manfredotti, M. Marinelli, G. Pucella, G. Verona-Rinati, Diamond Relat. Mater. 15, 797 (2006).

[3] C.M. Buttar, J. Conway, R. Meyfarth, G. Scarsbrook, P.J. Sellin, A. Whitehead, http://pppa.group.shef .ac.uk/reports/ 9703.pdf .

[4] W.U. Laub, T.W. Kaulich, F. Nusslin, Phys. Med. Biol. 44, 2183 (1999).

[5] G. Ciancaglioni, I. Consorti, R. Rossi, M.C. Conte, in: Nuclear Science Symp. Conf. Record, Vol. 7, IEEE, Rome 2004, p. 4445.
[6] G. Conte, M. Girolami, S. Salvatori, Appl. Phys. Lett. 91, 183515 (2007), doi: 10.1063/1.2805221, http://link. aip.org/link/?APPLAB/91/183515/1 .

[7] R.P. Hugtenburg, K. Johnston, G.J. Chalmers, A.H. Beddoe, Phys. Med. Biol. 46, 2489 (2001).

[8] R.D. Ilić, FOTELP-2K6, Photons, Electrons and Positrons Transport in $3 D$ by Monte Carlo Techniques, IAEA1388, http://www.nea.fr/abs/html/ iaea1388.html .

[9] S. Han, Rev. Sci. Instrum. 68, 647 (1997). 